\title{
Translating Probability Density Functions: From R to BUGS and Back Again
}

\author{
by David S. LeBauer, Michael C. Dietze, Benjamin M. Bolker
}

\begin{abstract}
The ability to implement statistical models in the BUGS language facilitates Bayesian inference by automating MCMC algorithms. Software packages that interpret the BUGS language include OpenBUGS, WinBUGS, and JAGS. R packages that link BUGS software to the R environment, including rjags and R2WinBUGS, are widely used in Bayesian analysis. Indeed, many packages in the Bayesian task view on CRAN (http://cran.r-project.org/web/views/Bayesian. html) depend on this integration. However, the R and BUGS languages use different representations of common probability density functions, creating a potential for errors to occur in the implementation or interpretation of analyses that use both languages. Here we review different parameterizations used by the R and BUGS languages, describe how to translate between the languages, and provide an $\mathrm{R}$ function, r2bugs.distributions, that transforms parameterizations from R to BUGS and back again.
\end{abstract}

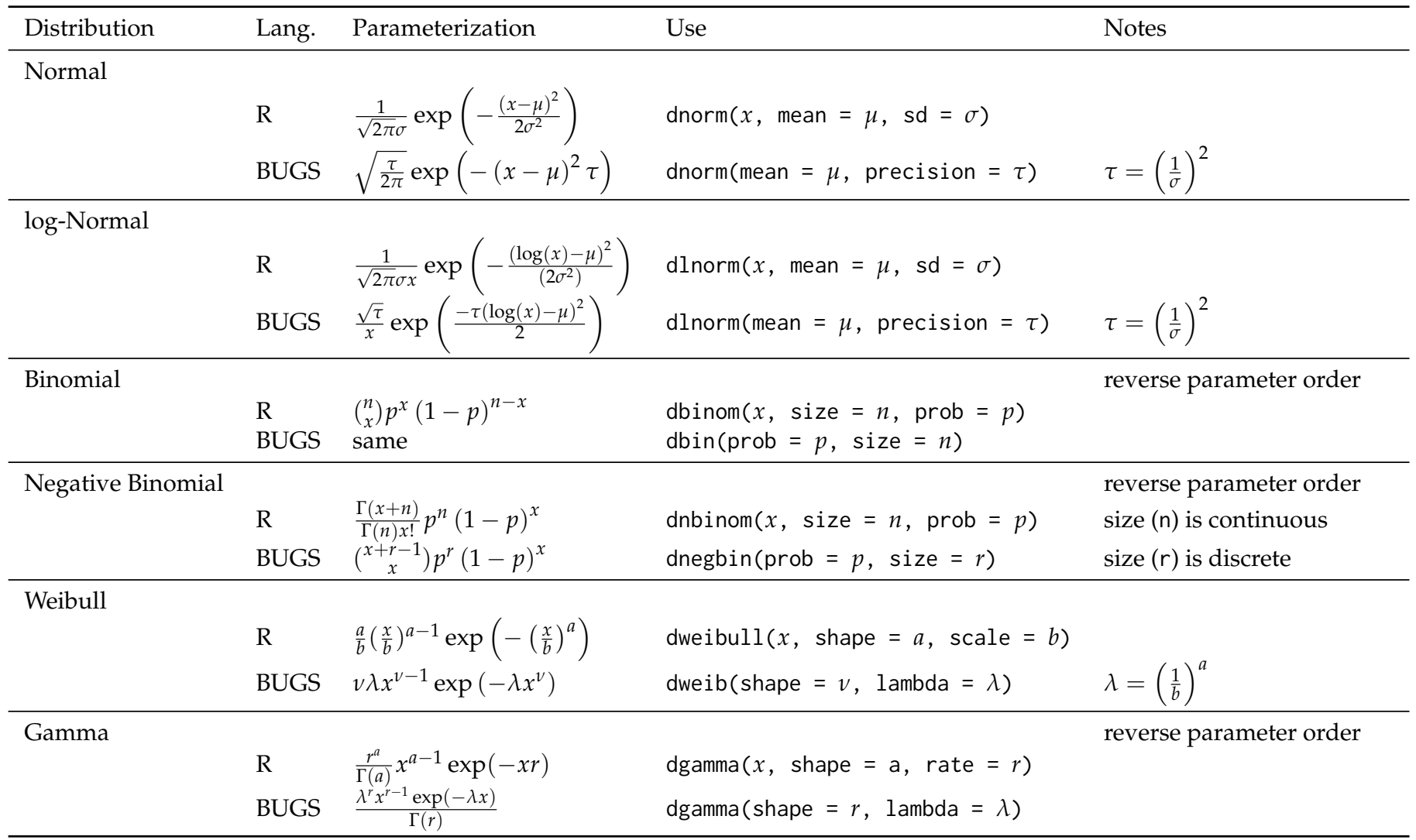

Table 1: Summary of different parameterizations of common distributions used by R and BUGS. Note: For ease of reference, parameterizations follow the JAGS and R documentation; as a result, the table includes equivalent equations that appear different, either because JAGS and R use different names for the same parameter or because the equation has been rearranged. For example, the shape parameter of the Gamma distribution is $r$ in the BUGS documentation and $a$ in the R documentation. For the Binomial, Negative Binomial, and Gamma distributions, BUGS and R expect parameters in different order (the order of parameters matters since arguments are assigned based on position in BUGS and may be in $\mathrm{R}$ as well). $\mathrm{R}$ allows alternate parameterizations for the Negative Binomial and Gamma distributions, but these are not shown here. The variable $x$ is implicit in all of the BUGS "Use" expressions. The Beta, Poisson, Exponential, and Uniform distributions have identical parameterizations in R and BUGS. 


\section{Probability density functions in R and BUGS}

R and BUGS implement many of the same probability distribution functions, but they often parameterize the same distribution differently (Table 1). Although these probability distribution functions are clearly described in the documentation of their respective languages, we were unable to find a summary of these differences in one place. The motivation for this article is to document and clarify these differences. Our sources are the JAGS documentation (Plummer, 2010) and the documentation of individual $\mathrm{R}$ functions.

\section{A bilingual translation function}

To support the automation of model specification in JAGS with priors computed and stored in R (LeBauer et al., 2013), we developed a function to translate parameterizations of common probability distributions from R to BUGS (and back again, by specifying direction = 'bugs $2 r$ '). Parameter transformations, parameter order, and differences in function names are documented in Table 1 and implemented in the $\mathrm{R}$ function r2bugs.distributions.

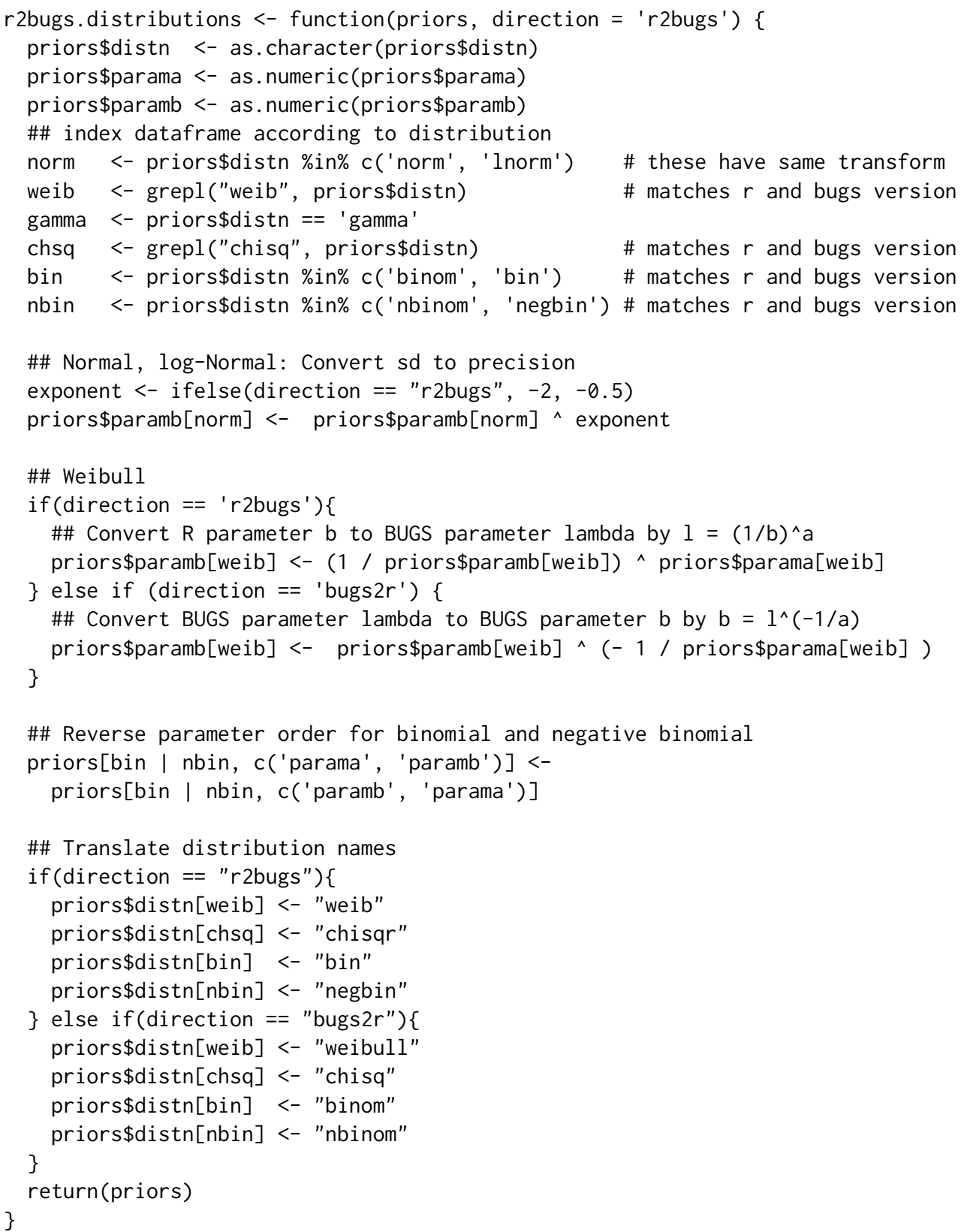




\section{A simple example}

As an example, we take the R-parameterized prior distribution $X \sim \mathcal{N}(\mu=10, \sigma=2)$ and convert it to BUGS parameterization $X \sim \mathcal{N}(\mu=10, \tau=1 / 4)$. We specify a model in JAGS that allows us to sample directly from a prior distribution. The function works for each of the distributions in Table 1. This particular example is the JAGS implementation of 'rnorm $(10000,10,2)$ ' in R. It is presented as minimal demonstration; for a non-trivial application, see LeBauer et al. (2013).

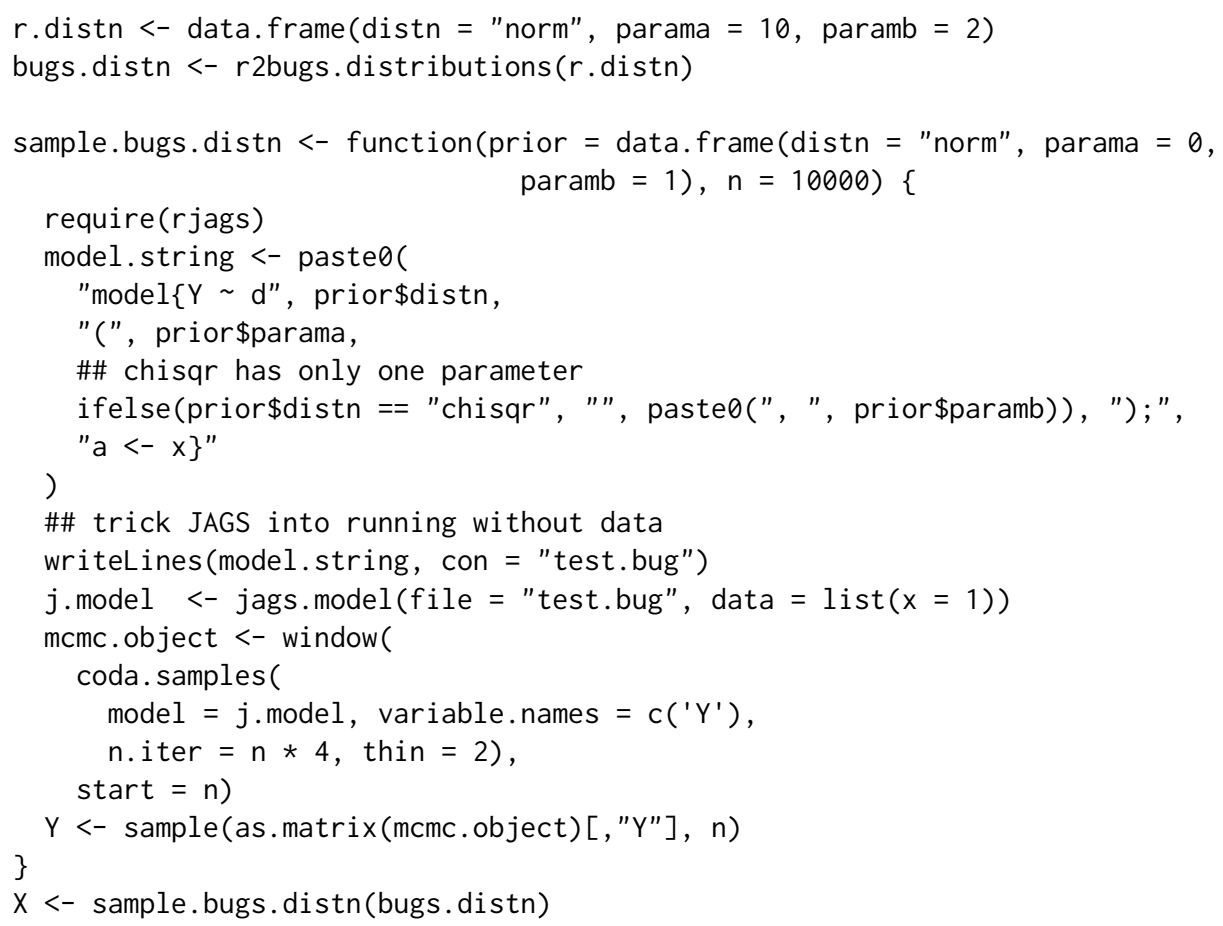

\section{Acknowlegements}

This collaboration began on the Cross Validated statistical forum (http://stats. stackexchange. com/ q/5543/1381). Funding was provided to DSL and MCD by the Energy Biosciences Institute.

\section{Bibliography}

D. S. LeBauer, D. Wang, K. T. Richter, C. C. Davidson, and M. C. Dietze. Facilitating feedbacks between field measurements and ecosystem models. Ecological Monographs, 83(2):133-154, 2013. URL http://www. esajournals.org/doi/abs/10.1890/12-0137.1. [p208, 209]

M. Plummer. JAGS Version 3.1.0 user manual, 2010. URL http://sourceforge. net/projects/mcmcjags/. [p208]

David S. LeBauer

Energy Biosciences Institute

University of Illinois

USA

dlebauer@illinois.edu

Michael C. Dietze

Department of Earth And Environment

Boston University

USA
Benjamin M. Bolker

Department of Mathematics \& Statistics

McMaster University

Canada 